Annuaire suisse de politique de développement

18 | 1999

La Suisse et l'action humanitaire

\title{
8. Coopération avec les pays en développement
}

\section{Gérard Perroulaz}

\section{OpenEdition \\ Journals}

Édition électronique

URL : http://journals.openedition.org/aspd/709

DOI : 10.4000/aspd.709

ISSN : 1663-9669

\section{Éditeur}

Institut de hautes études internationales et du développement

\section{Édition imprimée}

Date de publication : 1 janvier 1999

Pagination : 219-246

ISSN : 1660-5934

\section{Référence électronique}

Gérard Perroulaz, «8. Coopération avec les pays en développement », Annuaire suisse de politique de développement [En ligne], 18| 1999, mis en ligne le 17 juillet 2012, consulté le 07 septembre 2020 URL : http://journals.openedition.org/aspd/709 ; DOI : https://doi.org/10.4000/aspd.709 


\section{COOPÉRATION AVEC LES PAYS EN DÉVELOPPEMENT*}

\subsection{EVOLUTION GÉNÉRALE DE L'AIDE PUBLIQUE AU DÉVELOPPEMENT}

Le Comité d'aide au développement (CAD) est l'organe de l'OCDE chargé de traiter des questions de coopération au développement. Les pays membres se consultent pour examiner le volume et la nature de leurs contributions. Ils examinent périodiquement (tous les trois ans) les programmes d'aide de chacun des pays membres et se concertent également pour élaborer des stratégies communes dans le domaine de la coopération.

\section{$\square$ Contexte de baisse de l'aide des pays du CAD}

L'aide publique au développement (APD) totale a fortement baissé ces dernières années. Elle est passée de 60,9 milliards de dollars en 1992 à 55,4 milliards en 1996 et 47,6 milliards de dollars en 1997 (voir tableau no 18 ). Les Nations Unies recommandent aux pays donateurs d'affecter $0,7 \%$ de leur produit national brut (PNB) à l'aide publique au développement, mais seuls le Danemark, la Norvège, les Pays-Bas et la Suède se maintiennent en dessus de cet objectif. L'APD de tous les pays du CAD ne représentait en 1997 plus que $0,22 \%$ du PNB, soit le pourcentage le plus faible jamais enregistré depuis que l'objectif de $0,7 \%$ a été fixé. La moyenne pondérée des pays du CAD se situait encore à $0,33 \%$ du PNB en 1992 et l'APD s'est maintenue jusqu'au début des années 90 pendant deux décennies aux alentours de $0,35 \%$ du PNB.

\section{Aide publique aux pays en développement et aide aux pays en transition , Modifications récentes dans la liste des pays bénéficiaires de l'aide}

Le CAD distingue depuis 1994 deux listes de pays: les pays en développement et les pays en transition*. L'aide fournie aux pays et territoires en développement est comptabilisée dans l'agrégat d'aide publique au développement. Certains pays de l'Europe centrale et orientale étant compris dans cette liste de pays, I'aide apportée à ces pays peut donc être inclue dans I'APD: Albanie, Etats issus de l'ex-Yougoslavie, Etats de l'Asie centrale (Arménie, Azerbaïdjan, Kazakhstan, Kirghizistan, Ouzbékistan, Tadjikistan, Turkménistan), Géorgie, Moldavie (transférée dans la liste des pays en développement dès 1997).

Une deuxième liste de pays comprend les pays et territoires en transition: pays de l'Europe centrale et orientale, pays de la Communauté des Etats indépendants, mais aussi, ce qui est nouveau depuis quelques années, certains pays en développement plus avancés** . L'aide à ces pays n'est pas comprise dans I'APD, mais dans un agrégat séparé intitulé "aide publique aux pays en transition". Nouveauté importante dès 1997, I'aide à Israël ne peut plus être comptabilisée dans I'APD (alors que l'aide aux zones sous administration palestinienne est comptée dans I'APD). II convient donc toujours de tenir compte de ces changements lorsque l'on compare l'évolution de l'APD depuis le début des années 90. Par exemple, deux tiers de la baisse de l'APD des Etats-Unis entre 1996 et 1997 peuvent être expliqués par le retrait d'Israël de la liste des pays en développement.

ASTM 1999, I'aide aux pays en transition est détaillée dans le chapitre 9 de la partie Revue.

* Les deux listes complètes des bénéficiaires de l'aide établies par le CAD sont reproduites à la fin de la Revue, p. 261.

** Liste des pays et territoires en développement plus avancés dont l'aide ne peut plus être comptabilisée dans I'APD dès 1997: Bahamas, Bermudes, Brunei, îles Cayman, Chypre, Emirats arabes unis, îles Falkland, Hong Kong, Israël, Koweït, Qatar, Singapour, Taïwan.

\footnotetext{
* Par Gérard Perroulaz, chargé de recherche à l'IUED.
} 
Tableau $n^{\circ}$ 18: Evolution de l'aide publique

au développement des pays du CAD en \% du PNB

\begin{tabular}{lrrrrrrl}
\hline & $\mathbf{1 9 9 2}$ & $\mathbf{1 9 9 3}$ & $\mathbf{1 9 9 4}$ & $\mathbf{1 9 9 5}$ & $\mathbf{1 9 9 6}$ & $\mathbf{1 9 9 7}$ & Tendance \\
\hline Australie & 0.37 & 0.35 & 0.34 & 0.36 & 0.30 & 0.28 & Baisse \\
\hline Autriche & 0.30 & 0.30 & 0.33 & 0.33 & 0.24 & 0.26 & Baisse \\
\hline Belgique & 0.39 & 0.39 & 0.32 & 0.38 & 0.34 & 0.31 & Baisse \\
\hline Canada & 0.46 & 0.45 & 0.43 & 0.38 & 0.32 & 0.36 & Baisse \\
\hline Danemark & 1.02 & 1.03 & 1.03 & 0.96 & 1.04 & 0.97 & Stable \\
\hline Finlande & 0.64 & 0.45 & 0.31 & 0.32 & 0.34 & 0.33 & Forte baisse \\
\hline France & 0.63 & 0.63 & 0.64 & 0.55 & 0.48 & 0.45 & Baisse \\
\hline Allemagne & 0.39 & 0.36 & 0.34 & 0.31 & 0.33 & 0.28 & Baisse \\
\hline Irlande & 0.16 & 0.20 & 0.25 & 0.29 & 0.31 & 0.31 & Augmentation \\
\hline Italie & 0.34 & 0.31 & 0.27 & 0.15 & 0.20 & 0.11 & Forte baisse \\
\hline Japon & 0.30 & 0.27 & 0.29 & 0.28 & 0.20 & 0.22 & Baisse \\
\hline Luxembourg & 0.26 & 0.35 & 0.40 & 0.36 & 0.44 & 0.50 & Augmentation \\
\hline Pays-Bas & 0.86 & 0.82 & 0.76 & 0.81 & 0.81 & 0.81 & Baisse \\
\hline Nouvelle-Zélande & 0.26 & 0.25 & 0.24 & 0.23 & 0.21 & 0.25 & Fluctuant \\
\hline Norvège & 1.16 & 1.01 & 1.05 & 0.87 & 0.85 & 0.86 & Baisse \\
\hline Portugal & 0.36 & 0.28 & 0.34 & 0.25 & 0.21 & 0.25 & Baisse \\
\hline Espagne & 0.27 & 0.28 & 0.28 & 0.24 & 0.22 & 0.23 & Baisse \\
\hline Suède & 1.03 & 0.99 & 0.96 & 0.77 & 0.84 & 0.76 & Baisse \\
\hline Suisse & 0.39 & 0.34 & 0.36 & 0.34 & 0.34 & 0.32 & Baisse * \\
\hline Royaume-Uni & 0.31 & 0.31 & 0.31 & 0.29 & 0.27 & 0.26 & Baisse \\
\hline Etats-Unis & 0.20 & 0.15 & 0.14 & 0.10 & 0.12 & 0.08 & Forte baisse*** \\
\hline Moyenne pondérée & & & & & & & \\
des pays du CAD & $\mathbf{0 . 3 3}$ & $\mathbf{0 . 3 0}$ & $\mathbf{0 . 3 0}$ & $\mathbf{0 . 2 7}$ & $\mathbf{0 . 2 5}$ & $\mathbf{0 . 2 2}$ & \\
\hline En milliards de \$ & 60.85 & 56.49 & 59.15 & 58.93 & 55.49 & 47.58 & \\
\hline
\end{tabular}

Sources: rapport du CAD 1997 Coopération pour le développement. Efforts et politiques des membres du Comité d'aide au développement, Paris, OCDE, 1998 (pour les données de 1992 à 1996) ; Communiqué de presse de l'OCDE, Paris, 18.6.98 (pour les données provisoires concernant l'année 1997).

* La baisse de 1996 à 1997 est due à un changement du système de comptes nationaux, qui a entraîné une révision à la hausse du PNB et de ce fait une baisse du ratio APD/PNB.

* L'APD de la Suisse a été particulièrement élevée en 1992 en raison de l'adhésion aux institutions de Bretton Woods.

*** Deux tiers de la baisse de l'APD des Etats-Unis entre 1996 et 1997 peuvent être expliqués par le retrait d'Israël de la liste des pays en développement, car Israël était le principal pays bénéficiaire de l'aide américaine.

Le tableau $\mathrm{n}^{\circ} 18$ montre l'évolution de l'aide en pourcentage du PNB depuis 1992. On peut relever plusieurs aspects dans cette évolution:

- Depuis 1992, mis à part l'Irlande, le Luxembourg, la Nouvelle-Zélande et le Danemark, tous les pays ont connu une baisse de l'APD. Trois pays ont très fortement baissé leur aide: l'Italie, les Etats-Unis et la Finlande. En Autriche, aux Pays-Bas, en Espagne et au Royaume-Uni, la baisse a été relativement plus modérée.

- Les réductions des budgets consacrés à l'aide dans les pays du G7 expliquent quasiment toute la diminution récente de l'APD totale des pays du CAD. Parmi les pays du G7, seuls le Canada et le Japon ont augmenté leur APD en termes réels. Le total de l'APD fournie par les autres pays que ceux du G7 est resté assez stable ces dernières années.

- Les versements d'aide effectués par certains pays sont devenus plus irréguliers d'une année à l'autre, notamment en raison des décaissements irréguliers des contributions multilatérales. La forte diminution des contributions aux organismes multilatéraux explique par exemple la brutale baisse de l'aide du 
Japon et de la France entre 1995 et 1996 ainsi que le niveau particulièrement bas de l'APD du Canada, des Etats-Unis et de l'Italie en 1995.

口 L'aide de certains petits pays a beaucoup augmenté depuis 1992 (Irlande, Luxembourg).

- Une des raisons principales de la baisse de l'APD a été la volonté de diminuer les déficits budgétaires dans plusieurs pays, notamment dans les pays européens pour répondre aux critères de Maastricht. Les pays qui accusaient les déficits budgétaires les plus importants ont été les plus prompts à couper leurs budgets d'aide.

Le financement par l'aide au développement reste toutefois important pour certains pays, même si les apports privés (investissements directs et investissements de portefeuille) ont beaucoup augmenté ces dernières années (baisse en 1997 suite à la crise asiatique). Les apports privés ont passé de 77,3 milliards de dollars en 1992 à 286,3 milliards de dollars en 1996. Ces apports se concentrent pourtant sur un nombre très restreint de pays, et d'autres régions, demeurant à l'écart de ces flux, restent par conséquent très dépendantes des ressources financières telles que l'APD. Par exemple, l'Afrique subsaharienne a reçu en moyenne 27 dollars par habitant en aide et 3 dollars par habitant en investissements directs étrangers ${ }^{1}$. Les destinations principales des apports de capitaux privés restent les économies les plus dynamiques d'Asie, d'Europe et d'Amérique latine.

\section{$\square$ Aide aux pays les moins avancés (PMA)}

L'aide fournie aux PMA reste bien en deçà de l'objectif, fixé par la communauté internationale, d'une aide à ces pays représentant $0,15 \%$ au moins du PNB. La moyenne pour les pays du CAD se situait en 1996 à 0,05\% du PNB seulement. Le Danemark, la Norvège, la Suède et les Pays-Bas dépassent largement 1'objectif fixé (avec respectivement 0,33\% pour les deux premiers pays, 0,24 et $0,23 \%$ de l'aide destinée aux PMA). Les pays en dessous de la moyenne du CAD sont les Etats-Unis (0,02\% seulement de l'aide pour les PMA), le Japon, l'Autriche et la Nouvelle-Zélande. La Suisse consacrait en 1996 0,10\% de son aide pour les PMA².

$\square$ Volume de l'aide publique au développement et évolution récente pour la Suisse

La Suisse n'a jamais souscrit à l'objectif des Nations Unies d'une aide à 0,7\% du PNB. Au début des années 90, et notamment lors de la Conférence de Rio sur l'environnement et le développement, le Conseil fédéral avait exprimé à plusieurs reprises l'objectif d'atteindre progressivement une aide s'élevant à $0,4 \%$ du PNB. Compte tenu de l'état des finances fédérales et des coupures budgétaires adoptées par le Parlement, la Suisse s'éloigne cependant de cet objectif, puisque l'aide a passé ces dernières années de 0,36 en 1994 à 0,32\% du PNB en 1997 et que les perspectives restent sombres pour les années à venir. Selon les coupures budgétaires qui peuvent encore être décidées par le Parlement, l'APD pourrait baisser à $0,30 \%$ ou $0,29 \%$ du PNB d'ici la fin du siècle.

1. Source: Communiqué de presse de l'OCDE, Paris, 18.6.98.

2. Source: Communiqué de presse de l'OCDE, Paris, 15.6.98. 
L'APD fournie par la Suisse s'est élevée à 1217 millions de francs (1193,8 millions financés par la Confédération et 23,2 millions de francs d'aide fournie par les cantons et communes). Elle représente $3 \%$ des dépenses totales de la Confédération.

\subsection{ORGANISATION ET GESTION DE L'AIDE SUISSE}

\section{$\square$ Gestion de l'aide au sein de l'administration fédérale}

Deux offices de l'administration fédérale se répartissent la gestion de la plus grande partie de l'aide publique au développement. La Direction du développement et de la coopération (DDC), relevant du Département fédéral des affaires étrangères (DFAE), a géré près de 82\% de l'APD en 1997 (la coopération technique, une partie de l'aide financière, l'aide humanitaire). L'Office fédéral des affaires économiques extérieures (OFAEE), relevant du Département fédéral de l'économie, a géré 10\% de l'APD en 1997 (mesures de politique économique et commerciale). L'OFAEE gère en outre la plus grande partie de l'aide publique destinée aux pays de l'Europe centrale et orientale. Des représentants de l'OFAEE et de la DDC sont présents dans la plupart des délégations auprès de conférences internationales. D'autres départements et offices sont impliqués dans certains secteurs de la coopération internationale: Office fédéral de la santé publique pour l'OMS, Département fédéral des finances pour le FMI, Office fédéral de l'environnement pour les organisations et conventions internationales dans ce domaine, Office fédéral de l'éducation et de la science pour les bourses d'études, etc.

Le Conseil fédéral a décidé en mai 1998 de procéder à une fusion de l'OFAEE et de l'Office fédéral du développement économique et de l'emploi (OFDE, anciennement OFIAMT), afin de rassembler sous un même toit toutes les compétences du domaine économique (le nouvel office deviendra opérationnel d'ici l'an 2000). Les aspects de politique économique intérieure et extérieure seront ainsi intégrés. C'est le nouveau secrétaire d'Etat nommé par le conseiller fédéral Couchepin fin novembre 1998, David Syz, successeur de Franz Blankart, qui devra mener à bien la fusion entre les deux offices et diriger le futur Office de l'économie.

\section{$\square$ Cadre de la politique de développement}

La politique suisse du développement et de la coopération repose sur trois piliers:

- La Loi fédérale sur la coopération au développement et l'aide humanitaire internationale (du 19 mars 1976) et son ordonnance d'application (12 décembre 1977) - La loi spécifie que l'aide doit soutenir en priorité les efforts des pays en développement, régions et groupes de population les plus défavorisés.

- Les crédits-cadres pluriannuels présentés dans des «messages du Conseil fédéral» aux Chambres fédérales - Le Conseil national et le Conseil des Etats doivent approuver pour chaque instrument de coopération des engagements financiers sur plusieurs années (au maximum tous les quatre ans). Ces documents permettent de faire le bilan des expériences passées et de fixer les orientations de l'aide pour les années à venir. Le Parlement fixe ensuite les 
montants annuels de l'aide dans le cadre de l'adoption du budget annuel de la Confédération. Le tableau $\mathrm{n}^{\circ} 19$ donne la liste des principaux crédits de programme actuellement en vigueur.

- Les documents fixant les grandes orientations de l'aide - Les Lignes directrices Nord-Sud (adoptées en 1994) établissent les quatre objectifs de la politique de développement: 1) sauvegarder et maintenir la paix et la sécurité, promouvoir les droits de l'homme, la démocratie et l'Etat de droit; 2) promouvoir la prospérité; 3) améliorer la justice sociale; 4) protéger l'environnement naturel. Le document relève notamment la nécessité d'une meilleure cohérence entre la politique de coopération et les autres volets de la politique ainsi que les relations extérieures de la Suisse avec les pays en développement. L'idée est de mieux cerner les contradictions qui peuvent exister entre la coopération au développement et d'autres politiques (politique agricole, migration, environnement, politique d'encouragement à l'exportation). Sur cette base, la DDC et l'OFAEE peuvent donner leur avis sur des questions qui ne concernent pas stricto sensu la coopération, mais qui peuvent avoir des implications sur celle-ci, telles que les exportations d'armes, la lutte contre la corruption et les fuites de capitaux, l'accès au marché suisse pour des produits des pays en développement, la formulation d'un modèle de développement durable pour la Suisse, etc.

Tableau n¹9: Derniers crédits-cadres approuvés

\begin{tabular}{|c|c|c|c|}
\hline $\begin{array}{l}\text { Date du message } \\
\text { ( } n^{\circ} \text { de commande } \\
\text { du message) }\end{array}$ & Crédit-cadre & $\begin{array}{l}\text { Montant en } \\
\text { nillions de } \\
\text { rancs }\end{array}$ & $\begin{array}{l}\text { Durée minimale } \\
\text { et entrée } \\
\text { en vigueur }\end{array}$ \\
\hline $\begin{array}{l}21.2 .90 \\
(90.015)\end{array}$ & $\begin{array}{l}\text { Mesures de politique } \\
\text { économique et commerciale }\end{array}$ & 840 & $\begin{array}{l}4 \text { ans } \\
\text { de } 91 \text { à } 96\end{array}$ \\
\hline $\begin{array}{l}30.1 .91 \\
(90.080)\end{array}$ & $\begin{array}{l}\text { Financement de mesures } \\
\text { de désendettement en faveur } \\
\text { de pays en développement et } \\
\text { programmes en faveur de } \\
\text { l'environnement global }\end{array}$ & 700 & Dès 1991 \\
\hline 15.5 .91 & $\begin{array}{l}\text { Adhésion de la Suisse } \\
\text { aux institutions de Bretton Woods }\end{array}$ & 4986 & dès le 17.5 .92 \\
\hline $\begin{array}{l}20.4 .94 \\
(94.029)\end{array}$ & $\begin{array}{l}\text { Coopération technique } \\
\text { et aide financière }\end{array}$ & 3800 & $\begin{array}{l}4 \text { ans } \\
\text { dès le } 1.6 .95\end{array}$ \\
\hline $\begin{array}{l}31.5 .95 \\
(95.041)\end{array}$ & $\begin{array}{l}\text { Participation au capital des banques } \\
\text { régionales de développement }\end{array}$ & 800 & dès le 1.1 .96 \\
\hline $\begin{array}{l}13.12 .96 \\
(96.044)\end{array}$ & $\begin{array}{l}\text { Réorientation des mesures de } \\
\text { politique économique et commerciale }\end{array}$ & 960 & $\begin{array}{l}4 \text { ans } \\
\text { dès le } 1.1 .97\end{array}$ \\
\hline $\begin{array}{l}20.11 .96 \\
(96.092)\end{array}$ & Aide humanitaire internationale & 1050 & $\begin{array}{l}4 \text { ans } \\
\text { dès le } 1.2 .98\end{array}$ \\
\hline 7.12 .98 & $\begin{array}{l}\text { Coopération technique } \\
\text { et aide financière }\end{array}$ & 4000 & $\begin{array}{l}4 \text { ans } \\
\text { De } 99 \text { à } 02\end{array}$ \\
\hline
\end{tabular}

Sources: DDC, OFAEE.

Le Conseil fédéral a présenté en décembre 1998 son nouveau message concernant la continuation de la coopération technique et de l'aide financière, crédit de programme de 4 milliards de francs suisses pour les années 1999-2002. Le message permet non seulement de définir le cadre financier, mais de faire le bilan des activités passées et de donner les grandes orientations de l'aide pour les années à venir (l'Annuaire 2000 reviendra en détail sur le contenu du nouveau message). 


\section{$\square$ Rapport sur dix ans de coopération internationale}

En réponse au postulat de la conseillère nationale Rosmarie Zapfl-Helbling de septembre 1996, le Conseil fédéral a publié en 1998 un rapport sur la coopération internationale de la Suisse avec les pays du Sud et de l'Est pour les années 1986-1995. Le rapport rappelle les mutations intervenues dans la politique mondiale et les nouveaux défis pour la coopération internationale. Il fait aussi le bilan des adaptations de la coopération suisse à ces changements et des contributions qu'elle cherche à apporter pour résoudre certains problèmes mondiaux.

Parmi les grandes mutations globales de la période 1986-1995, le rapport relève la différenciation des situations dans le monde: situation des pays nouvellement industrialisés, problèmes des pays les moins avancés (PMA) et surtout processus de transition économique et politique dans l'ancien bloc de l'Est. Si la justice sociale et la pauvreté restent des problèmes de base, une attention plus grande a été portée aux questions de la protection du cadre de vie naturel et à l'avènement de sociétés plus démocratiques. Les gouvernements et l'opinion publique ont pris de plus en plus clairement conscience que certains problèmes transfrontaliers (changements climatiques, migrations, prolifération des armes, criminalité internationale, drogues) représentent des risques beaucoup plus graves pour leur pays que l'éventualité d'agressions militaires plus classiques. La notion de la «sécurité humaine globale» repose sur l'interdépendance entre les pays et sur le fait que les pays industrialisés et les pays en développement ont un intérêt vital commun à résoudre certains problèmes mondiaux.

Plusieurs aspects gagnent en importance dans le cadre de la coopération internationale: prise en compte de la nécessité d'une bonne gestion des affaires publiques $^{3}$, démocratisation et participation politique plus ouverte à la société civile, prise en compte de la condition des femmes, environnement et développement.

Le rapport relève une série d'instruments de coopération que la Suisse a mis sur pied pour répondre à certains défis. Quelques approches de la coopération suisse sont originales et novatrices: souci de cohérence entre les politiques de coopération et les autres politiques de la Confédération envers les pays en développement, politique de désendettement (fonds de contrepartie, initiatives pour les pays les plus lourdement endettés). La coopération suisse attache en outre une grande importance au développement participatif (autopromotion, promotion des organisations de base) et à la collaboration avec les ONG d'entraide et les milieux de l'économie privée suisse.

Dans les conclusions générales sur la coopération internationale, le rapport réaffirme que la coopération est un investissement nécessaire dans notre avenir à tous et que les efforts en vue d'une amélioration qualitative de l'aide doivent être poursuivis. Il relève que la coopération suisse a prouvé qu'elle pouvait rester dynamique et souple face aux nouveaux défis, et souligne la complémentarité entre coopération multilatérale et bilatérale. Le rapport réaffirme aussi la nécessité d'une meilleure cohérence dans les relations avec les pays en développement, et que le développement humain doit rester la préoccupation première de la coopération suisse.

3. La notion de good governance, ou bonne gestion des affaires publiques, englobe les aspects suivants: encouragement des principes de l'Etat de droit, gestion efficace, lutte contre la corruption et réduction des dépenses militaires excessives. 


\subsection{EVOLUTION GÉNÉRALE DE L'AIDE BILATÉRALE}

L'aide bilatérale a baissé depuis 1994, en passant de 985 millions de francs en 1994 à 895 millions de francs en 1996 et 841 millions de francs en 1997. Les bases de travail pour orienter la coopération bilatérale relevant de la DDC sont les papiers de réflexion stratégiques par thèmes ou secteurs (politique de promotion de l'artisanat et de l'industrie, ou développement urbain par exemple), les programmes par pays (PPP, programmes sur cinq ans établis par la DDC et l'OFAEE) et les programmes annuels.

Le programme par pays établi pour chaque pays de concentration renseigne sur la situation et les besoins de l'Etat en question, et définit les objectifs généraux de la coopération suisse, les stratégies prévues et les ressources humaines et financières nécessaires pour atteindre ces objectifs. Les programmes annuels précisent les étapes de la mise en œuvre et permettent d'adapter les programmes par pays. Dans ces programmes, on vise aujourd'hui à une meilleure articulation entre les différents niveaux d'intervention: population locale bénéficiaire (niveau micro), cadre institutionnel (niveau méso) et appui politique (niveau macro).

Les bureaux de coordination (BUCO) assurent la présence décentralisée de la DDC auprès des partenaires dans les pays dits de concentration; ces BUCO jouent un rôle essentiel en étant proches des partenaires locaux et des réalités administratives des pays d'intervention de l'aide suisse.

La coopération suisse encourage l'appropriation locales des projets et programmes (tenir compte des besoins exprimés par les populations bénéficiaires de l'aide, travailler avec les organisations locales et la société civile, autopromotion, promotion d'organisations de base).

\section{$\square$ Pays de concentration de l'aide suisse}

La DDC poursuit une politique de concentration de l'aide sur certains pays. Les pays dits de concentration sont passés progressivement de 20 à 16. Les autres volets de la coopération au développement n'obéissent pas au critère de concentration sur les mêmes pays que la coopération technique de la DDC: le versement des bourses d'étude, l'aide humanitaire, les mesures financées par l'OFAEE et les contributions versées aux ONG ne se dirigent pas forcément surtout dans les pays dits de concentration. L'OFAEE, de par la nature de ses instruments (mesures de désendettement, aide à la balance des paiements, etc.), ne peut pas se concentrer géographiquement de la même manière que la DDC.

- Liste des pays ou régions de concentration de l'aide fournie par la DDC - Afrique: Bénin, Burkina Faso, Madagascar, Mali, Mozambique, Niger, Tanzanie, Tchad; Amérique latine: Bolivie, Nicaragua/Amérique centrale, Pérou; Asie: Bangladesh, Inde, Népal, Pakistan et Vietnam/région du Mékong.

- Liste des principaux pays d'intervention de l'OFAEE qui sont aussi des pays de concentration de la DDC: Bolivie, Burkina Faso, Inde, Mozambique, Nicaragua, Pakistan, Pérou, Tanzanie, Vietnam.

- Autres principaux pays d'intervention de l'OFAEE (où la DDC est peu présente) : Chine, Egypte, Indonésie (pays de concentration de la DDC jusqu'en 1995), Ouganda. 
Le tableau $n^{\circ}$ 3.3.A. de la section «Statistiques» relève les montants de coopération technique et d'aide financière versés par la DDC dans les pays de concentration de la DDC. Les versements les plus élevés ont concerné l'Inde (28,1 millions de francs en 1997), l'Amérique centrale (21,5 millions) et la Tanzanie (20,5 millions). Dès 1997, le Vietnam a remplacé l'Indonésie dans la liste des pays de concentration. La coopération avec Madagascar s'est interrompue en 1998, notamment suite aux lenteurs de l'enquête sur l'assassinat, deux ans auparavant, d'un coopérant suisse.

\section{Répartition géographique de l'aide bilatérale}

Le graphique $\mathrm{n}^{\circ} 5$ montre les principaux pays bénéficiaires de l'aide bilatérale suisse en mettant en évidence les différences dans les types d'aide. Certains pays reçoivent beaucoup d'aide humanitaire (ex-Yougoslavie et Rwanda) ; d'autres sont d'importants partenaires pour la coopération au développement et les mesures de politique économique et commerciale représentent une grande part de l'aide apportée à certains pays (Bolivie, Burkina Faso, Mozambique et Tanzanie).

\section{Graphique $n^{\circ} 5$ : Principaux pays et régions bénéficiaires de l'aide suisse et répartition par type d'aide (Total cumulé de l'aide bilatérale, en millions de francs}

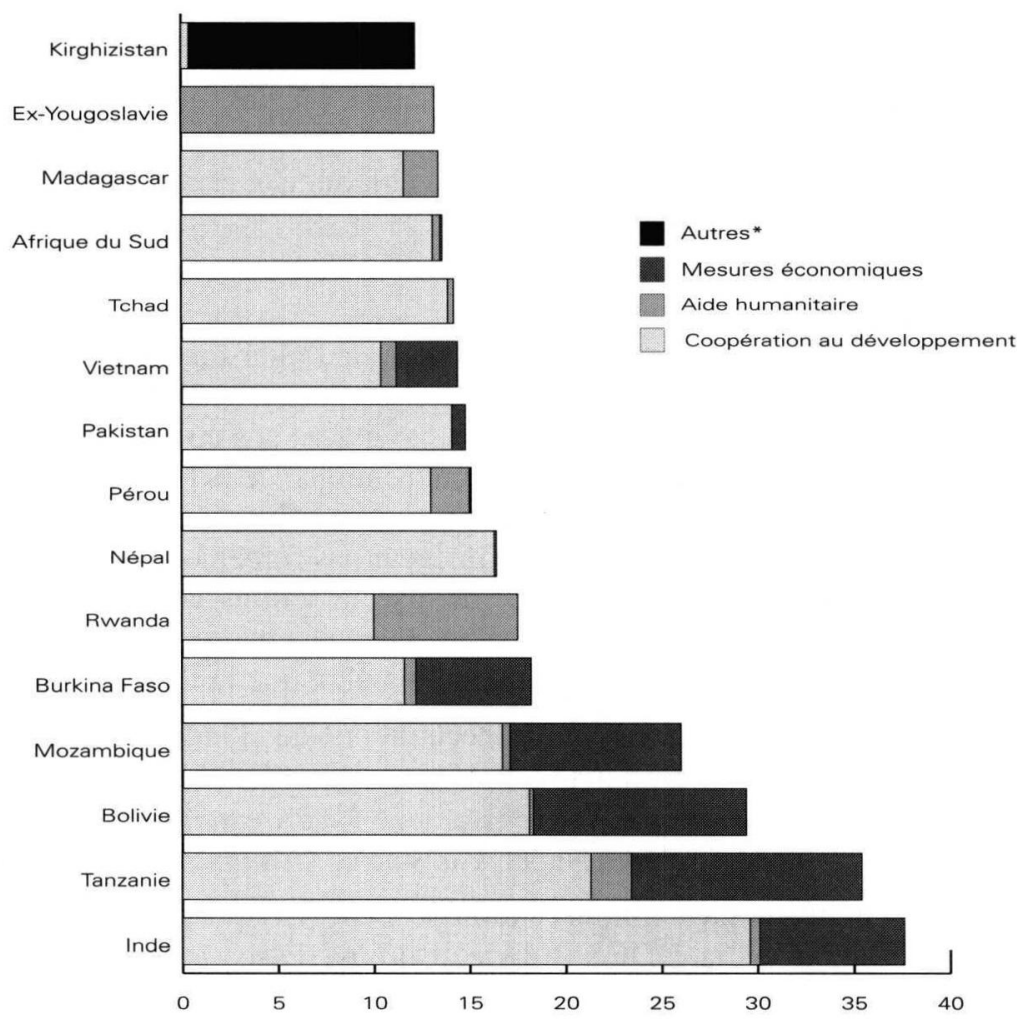

Source: graphique élaboré à partir du tableau $\mathrm{n}^{\circ} 3$ de Coopération internationale de la Suisse. Rapport annuel 1997 (brochure Coopération au développement), DDC/OFAEE, Berne, 1998.

* Les autres mesures comprennent par exemple l'aide financière. 


\section{$\square$ Répartition sectorielle de l'aide et thèmes prioritaires}

Les secteurs prioritaires de l'aide bilatérale sont l'agriculture et l'élevage (16\% de l'aide bilatérale versée par la DDC en 1997), l'infrastructure, l'eau et l'énergie (14\%), l'éducation, l'information et la culture (13\%), la forêt et l'environnement $(9 \%)$, et la santé, la nutrition et la population $(8 \%)$. Plusieurs thèmes reçoivent une attention particulière dans la gestion des projets de coopération: développement durable, développement équilibré homme/femme (gender), participation des populations concernées par l'aide. Au lieu de se limiter à faire des projets spécifiques dans l'un de ces domaines, ces thèmes devraient être pris en compte dans tous les programmes d'aide. Les politiques sectorielles sont régulièrement révisées par la DDC. L'OFAEE se concentre sur les domaines dans lesquels l'économie suisse a un avantage comparatif, ainsi que sur les réformes économiques, le transfert de technologies (en particulier pour la protection de l'environnement) et la mobilisation des ressources privées.

La DDC a établi en 1998 un inventaire des activités en milieu urbain. Le rapport montre que plus de 270 activités ont été réalisées de 1993 à 1998 en milieu urbain pour des projets représentant un tiers du budget bilatéral annuel de la DDC.

\section{$\square$ Promotion des droits humains}

Des lignes directrices sur le thème «Promotion des droits de l'homme et coopération au développement» ont été élaborées par la DDC et l'OFAEE en février $1997^{4}$. Le document rappelle les grandes lignes de la politique extérieure de la Confédération dans le domaine des droits humains, ainsi que les implications de cette politique dans la conduite des projets de coopération au développement. L'engagement en faveur des droits de l'homme fait partie des cinq objectifs prioritaires définis dans le rapport du Conseil fédéral sur la politique extérieure de la Suisse dans les années 90 . Les Lignes directrices Nord-Sud réaffirment cet engagement en le fixant comme l'un des objectifs stratégiques de l'action en faveur des pays en développement (sauvegarder et maintenir la paix et la sécurité, promouvoir les droits de l'homme, la démocratie et l'Etat de droit). Les lignes directrices sur la promotion des droits humains mettent en avant un certain nombre de principes à respecter dans la conduite des projets de coopération. La Confédération souhaite mettre l'accent sur des mesures positives et sur le dialogue plutôt que sur des sanctions. L'interruption de projets de coopération ou la suspension de la coopération avec un pays représenteront la solution ultime en cas de violations graves et systématiques des droits humains. Il est dans ce cas aussi possible de retirer l'aide aux organismes gouvernementaux pour la confier à des ONG. La pratique de la DDC va en fait plutôt dans le sens de mesures incitatives, avec des mesures visant à conscientiser la population, à soutenir les plus pauvres, à renforcer la société civile, à améliorer la législation, l'application du droit, la protection judiciaire et la formation, à promouvoir l'Etat de droit et à apporter une aide aux médias indépendants. Le document reconnaît qu'une politique crédible des droits de l'homme devrait aussi se traduire par une meilleure cohérence entre les différents instruments de la politique extérieure suisse. La cohérence est à rechercher entre la politique de coopération et les autres politiques de la Confé-

4. Les lignes directrices sont publiées dans le document de la DDC Promotion des droits de l'homme et coopération au développement, DDC, Berne, 1997, 24 p. Sur le thème des droits de l'homme, voir aussi le magazine de la DDC Un seul monde, $\mathrm{n}^{\circ} 4,1998$. 
dération, telles que la politique de migration et d'aide au retour, la politique de promotion des exportations et des investissements, les autorisations d'exporter du matériel de guerre. Pour l'OFAEE, il est aussi possible d'œuvrer en faveur des droits humains par une politique de libéralisation de l'économie des pays en développement, d'ouverture face à l'extérieur et d'intégration dans l'économie mondiale, en évitant ainsi un isolement complet d'un pays.

\subsection{AIDE BILATÉRALE - MESURES DE POLITIQUE ÉCONOMIQUE ET COMMERCIALE}

Ces instruments de coopération sont gérés par l'Office fédéral des affaires économiques extérieures (OFAEE). Le $5^{\mathrm{e}}$ crédit de programme pour la continuation et la réorientation des mesures de politique économique et commerciale est entré en vigueur au début de l'année 1997. L'OFAEE proposait une réorientation des mesures en mettant beaucoup plus l'accent sur la collaboration avec le secteur privé. Les entreprises privées ne sont plus seulement exécutrices de mandats, mais les projets de coopération sont de plus en plus développés et conçus en collaboration directe avec les entreprises privées. Les pays en développement sont encouragés dans leurs efforts pour s'intégrer dans l'économie mondiale et pour attirer les investissements.

ASTM 1997, «Nouveau message concernant le financement et la réorientation des mesures de politique économique et commerciale», pp. 152-165.

Le tableau $n^{\circ} 20$ récapitule les versements de l'OFAEE pour les différentes mesures de politique économique et commerciale de 1991 à 1998. L'importance donnée aux différents instruments d'aide a changé pendant ces huit dernières années. Les mesures de soutien dans le secteur des produits de base ont fortement diminué depuis 1991. Les mesures de promotion des investissements du secteur privé dans les pays en développement gagnent de l'importance, alors que les mesures visant à renforcer les conditions-cadres des pays en développement demeurent un pilier important de la coopération économique suisse.

Tableau n²0: Mesures de politique économique et commerciale Montants versés par instrument, de 1991 à 1998

\begin{tabular}{|c|c|c|c|c|c|c|c|c|c|}
\hline Types & & & & & Verser & nents & & & \\
\hline & mio. & $\% d u$ & & en & millions & de frar & & & mio. \% du \\
\hline & & 991 & 1992 & 1993 & 1994 & 1995 & 1996 & 1997 & 1998 \\
\hline $\begin{array}{l}\text { Aide à la balance } \\
\text { balance des paiements }\end{array}$ & 52.2 & 22.9 & 43.5 & 25.5 & 29.2 & 13.0 & 29.5 & 40.2 & 10.5 \\
\hline $\begin{array}{l}\text { Mesures } \\
\text { de désendettement }\end{array}$ & 80.0 & 35.1 & 58.9 & 48.9 & 41.4 & 68.4 & 38.1 & 21.2 & 40.426 .6 \\
\hline Promotion commerciale & 5.8 & 2.5 & 5.2 & 5.3 & 5.1 & 7.5 & 13.5 & 4.8 & 10.2 \\
\hline Produits de base & 16.9 & 7.4 & 11.8 & 8.1 & 3.5 & 7.3 & 3.3 & 3.6 & 3.4 \\
\hline $\begin{array}{l}\text { Promotion } \\
\text { des investissements }\end{array}$ & 4.1 & 1.8 & 3.1 & 2.9 & 23.0 & 9.3 & 21.0 & 20.0 & $61.0 \quad 40.2$ \\
\hline Financements mixtes & 67.7 & 29.6 & 83.3 & 87.4 & 75.4 & 26.9 & 27.7 & 10.0 & $22.1 \quad 14.6$ \\
\hline Etudes et administration & 1.5 & 0.7 & 2.3 & 2.8 & 2.5 & 3.4 & 2.6 & 4.1 & 4.8 \\
\hline $\begin{array}{l}\text { Total des } \\
\text { mesures économiques }\end{array}$ & 228.3 & 100.0 & 208.2 & 180.9 & 180.2 & 135.9 & 135.7 & 103.7 & 151.7100 .0 \\
\hline
\end{tabular}

Source: tableau élaboré sur la base de données fournies par l'OFAEE. 
L'aide financière gérée par l'OFAEE s'est élevée à 104 millions de francs en 1997 et à 152 millions de francs en 1998. Les financements mixtes ne représentent plus que $15 \%$ du total en 1998 , alors qu'ils représentaient entre un tiers et presque la moitié du volume total de l'aide économique de 1990 à 1994. Les mesures de promotion des investissements gagnent de l'importance ces dernières années, pour atteindre 40\% de l'aide en 1998.

\section{$\square$ Aide à la balance des paiements}

Cet instrument de coopération permet, dans certaines situations, de financer des importations de première nécessité en provenance de la Suisse (matériel médical et technique) et, dans un cas plus général, d'assister financièrement le gouvernement bénéficiaire dans la conduite d'un ajustement structurel par exemple. En 1997, la Suisse a conclu deux nouveaux accords d'aide à la balance des paiements avec le Sénégal (engagement de 13 millions de francs) et la Tanzanie (12 millions de francs, $4^{\mathrm{e}}$ aide financière de ce type), alors que des décaissements liés à des opérations en cours avaient lieu en Bolivie, au Burkina Faso, en Erythrée et au Mozambique. En 1998, un nouvel accord a été conclu avec le Mozambique (pays de concentration de la coopération suisse) portant sur une cinquième aide à la balance des paiements, pour un montant total de 18 millions de francs. Cette opération devrait durer deux ans et permettre ainsi au Mozambique de consolider son assainissement financier et de poursuivre son programme de réformes économiques et structurelles. Les versements de l'OFAEE en 1998 se sont élevés à 10,5 millions de francs, dont 8,3 millions pour le Mozambique.

\section{$\square$ Mesures de désendettement}

Les mesures de désendettement ont été introduites dès 1991 lors de l'adoption du crédit de programme du $700^{\mathrm{e}}$ anniversaire de la Confédération, suite à la pétition lancée par les œuvres d'entraide suisses en faveur du désendettement. Sur le total du crédit-cadre de 700 millions de francs, 400 millions ont été affectés au soutien des mesures de désendettement. Le programme suisse de désendettement a proposé des solutions originales et des pistes novatrices (avec les fonds de contrepartie par exemple). La Suisse a appuyé dans les institutions de Bretton Woods des dossiers pour le rachat des dettes multilatérales et pour le désendettement des pays pauvres lourdement endettés. Les versements liés aux mesures de désendettement se sont élevés à 40,4 millions de francs en 1998, dont 18 millions de francs pour les pays concernés par l'initiative HIPC, 7,4 millions de francs pour le Nicaragua et 5 millions de francs pour la Guinée-Bissau (ainsi que 8,3 millions pour d'autres pays africains).

ASTM 1999, dans le chapitre 1 de la «Revue», voir les parties 1.2 («Endettement extérieur») sur les mesures de désendettement prises sur le plan multilatéral et les mesures suisses et 1.4 («Institutions financières internationales») sur la politique de la Suisse au sein du FMI et de la Banque mondiale.

\section{$\square$ Promotion des investissements}

Le nouveau crédit de programme proposait une série de nouveaux instruments de coopération destinés à stimuler les exportations et les investissements vers les pays en développement:

- La nouvelle Société financière suisse pour le développement (Swiss Development Finance Corporation - SDFC) permettra de donner un appui aux 
entreprises de l'OCDE (en particulier les PME) qui souhaitent lancer des joint-ventures ou moderniser des entreprises existantes dans un pays en développement ou dans un pays de l'Europe orientale. La Société financière est une entreprise conjointe du secteur privé et du secteur public suisses. Le capital prévu de 100 millions de francs est souscrit à 51\% par des actionnaires privés et à $49 \%$ par la Confédération. La nouvelle SDFC devient opérationnelle dès le printemps 1999. Elle accorde un capital-risque pour les projets d'investissements directs et fournira des services (aide au management). Les décisions sont prises sur la base de la rentabilité du projet et de critères de politique de développement.

- Les autres mesures de promotion des investissements prévues dans le créditcadre comprennent des appuis pour la mise en relation d'affaires (entre entreprises du Nord et du Sud, voir la SOFI ci-dessous), le financement d'études pour des investissements et d'activités de formation du secteur privé, l'encouragement du transfert de technologies respectueuses de l'environnement.

Le programme de mise en relation d'affaires entre les entreprises suisses et celles des pays en développement et en transition est géré par la nouvelle fondation SOFI (Swiss Organization for Facilitating Investments). Cette fondation a été créée en juillet 1997 par l'OFAEE, dans le but de promouvoir les investissements suisses dans les pays en développement et dans les pays en transition. La SOFI fournit des informations sur les marchés potentiels (conditions-cadres dans certains pays, législation locale, mesures d'incitation des investissements, données sur les marchés du travail, la taxation, etc.). Elle apporte son aide pour la mise en relation entre partenaires (pour des joint-ventures ou d'autres projets de coopération à long terme) : recherche de partenaires potentiels, conseil, études de faisabilité, accompagnement et médiation durant la phase de négociation, assistance dans la préparation et la réalisation de projets. La SOFI doit permettre ainsi de favoriser le transfert de savoir-faire entre les entreprises suisses (surtout les PME) et celles des pays partenaires ${ }^{5}$.

La Confédération a aussi mis sur pied un fonds de capital-risque, le Swiss Technology Venture Capital Fund, destiné à financer des entreprises conjointes (jointventures), principalement en Inde. Le Fonds sino-suisse de partenariat a été créé en décembre 1997 pour soutenir les PME suisses qui souhaitent s'implanter dans le marché chinois.

En 1998, les principaux versements dans le domaine de la promotion des investissements ont concerné l'Inde (17,1 millions de francs), la Chine (10,8 millions) et l'île Maurice (10,5 millions). Les versements totaux se sont élevés à 61 millions de francs (contre 20 millions en 1997).

Ca ASTM 1999, dans la «Revue», voir le chapitre 7 «Politique économique extérieure» pour la promotion des investissements et du commerce.

\section{$\square$ Financements mixtes}

Les financements mixtes combinent un don de la Confédération et un prêt (aux conditions du marché) consenti par un consortium de banques suisses. Ces crédits permettent au pays partenaire d'acheter des biens d'équipement et des services en Suisse (pour 50\% au minimum du crédit) ou dans d'autres pays. Huit

5. Adresse de la SOFI: case postale, 8026 Zurich. Tél. 01/249.30.50; site Internet: http://www.sofi.ch. 
accords ont donné lieu à des déboursements en 1997 et 1998. L'importance de cet instrument est beaucoup moins grande qu'il y a quelques années, puisque les versements sur les financements mixtes en cours n'ont atteint que 10 millions de francs en 1997 (contre 75 à 87 millions de francs dans les années 1992-1994). La raison principale de cette baisse est à chercher dans la difficulté que rencontrent les entreprises suisses à identifier des projets conformes aux nouvelles règles de l'OCDE; les règles de conduite de l'OCDE restreignent le recours aux financements mixtes aux projets commercialement non viables. Les financements mixtes ont à nouveau occasionné des versements de 22,1 millions de francs en 1998, dont 8,8 millions pour l'Egypte, 4,4 millions pour la Jordanie, 3,6 millions pour la Chine, 1,6 pour la Tunisie et 1,3 pour le Vietnam.

Un accord portant sur un $4^{\mathrm{e}}$ financement mixte, d'un montant de 80 millions de francs, a été signé fin 1997 avec l'Egypte.

\subsection{AIDE HUMANITAIRE}

L'aide humanitaire peut être fournie par les canaux de l'aide bilatérale ou multilatérale. En 1997, les Chambres fédérales ont approuvé un nouveau crédit-cadre de 1,05 milliard de francs, pour une durée de quatre ans au moins (voir Annuaire 1998, pp. 324-327). Le crédit-cadre sur l'aide humanitaire concerne les pays en développement, mais aussi les pays de l'Europe centrale et orientale (l'aide à ces pays étant toutefois comprise non pas dans l'aide publique au développement, mais dans l'agrégat d'aide publique aux pays en transition). Chaque année, les crédits de paiement mis à disposition sont fixés par les Chambres fédérales dans le cadre du budget de la Confédération (156 millions de francs pour l'année 1997 et 158 millions de francs pour 1998). Un autre crédit-cadre règle les contributions ordinaires au budget siège du CICR (65 millions de francs pendant quatre ans). Un nouveau crédit-cadre pour le budget siège du CICR a été adopté par le Parlement en décembre 1997. Cette enveloppe budgétaire s'élève à 275 millions de francs pour les années 1998 à 2001.

L'aide humanitaire de la Confédération s'est élevée à 222,1 millions de francs en 1997, dont 217,1 millions comptabilisés dans l'APD (soit 17,8\% de l'APD totale). L'aide humanitaire est en baisse ces dernières années (230,4 millions en 1996, 253,7 millions en 1993). Les principaux pays bénéficiaires de l'aide humanitaire en 1997 étaient l'ex-Yougoslavie (13,2 millions de francs), l'Angola (8,3 millions), les républiques du Caucase ( 8,2 millions), le Rwanda $(7,5)$ et la Corée du Nord $(6,3)$.

Sur les 222 millions de francs d'aide, 72,2 millions de francs d'aide humanitaire ont été acheminés par les organisations internationales (dont 28 millions pour le HCR et 26 millions pour le Programme alimentaire mondial PAM), 81 millions par le CICR et 44 millions par le biais des œuvres d'entraide suisses. Les actions directes du Corps suisse d'aide en cas de catastrophe (ASC) se sont élevées à 25 millions de francs ${ }^{6}$. Le graphique $n^{\circ} 6$ montre cette répartition de l'aide humanitaire.

6. Voir la brochure Corps suisse d'aide en cas de catastrophe (ASC) éditée en 1998 par la DDC à l'occasion du $25^{\circ}$ anniversaire de la création de l'ASC. 


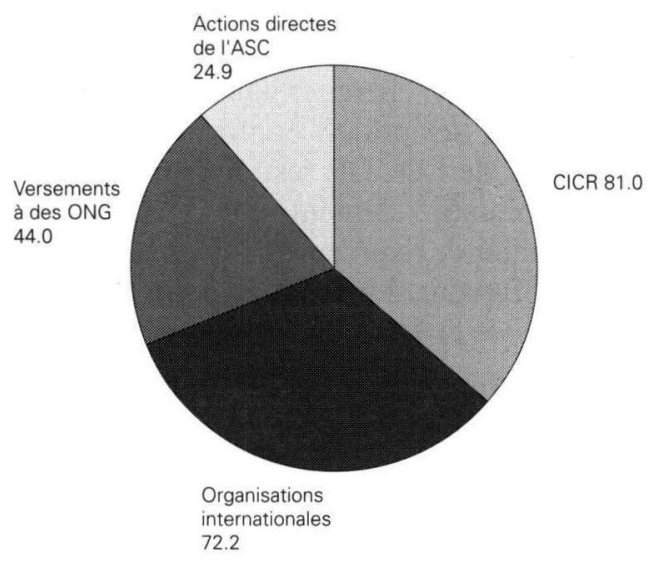

Source: DDC/OFAEE, Coopération internationale de la Suisse. Rapport annuel 1997 (brochure Aide humanitaire), Berne, 1998.

Le tableau n 21 montre l'évolution des contributions de la Suisse aux principales organisations multilatérales actives dans l'aide humanitaire. La fonction de coordination des actions humanitaires des Nations Unies, confiée autrefois au Département des affaires humanitaires (DAH, qui avait son siège à Genève), a été reprise dès 1998 par le nouveau Bureau du coordinateur de l'ONU pour les affaires humanitaires (dont le siège est à New York, avec une représentation à Genève). La Suisse est l'un des principaux bailleurs de fonds de cette institution de coordination (6,1 millions de francs versés en 1997). De 1992 à 1997, les contributions de la Suisse à l'ONU se sont réparties de la manière suivante: le HCR a reçu en moyenne $40 \%$ des moyens financiers versés par la Suisse, le PAM $39 \%$ de ces moyens, l'UNRWA (Office de secours et de travaux des Nations Unies pour les réfugiés de Palestine dans le Proche-Orient) $12 \%$ et le Département des affaires humanitaires $4 \%$ (plus $5 \%$ pour des organisations diverses).

Tableau n²1: Contribution de la Confédération aux organismes internationaux actifs dans l'aide humanitaire (1993-1997), en millions de francs

\begin{tabular}{lrrrrr}
\hline & $\mathbf{1 9 9 3}$ & $\mathbf{1 9 9 4}$ & $\mathbf{1 9 9 5}$ & $\mathbf{1 9 9 6}$ & $\mathbf{1 9 9 7}$ \\
\hline Organisations multilatérales & & & & & \\
\hline HCR & 43.2 & 31.9 & 28.4 & 29.1 & 27.4 \\
\hline PAM & 32.1 & 35.1 & 30.5 & 32.6 & 26.9 \\
\hline DAH Coordination aide humanitaire & 1.3 & 1.2 & 2.6 & 5.6 & 6.1 \\
\hline UNRWA & 12.7 & 10.6 & 8.7 & 9.5 & 7.7 \\
\hline Autres organisations (UNICEF, OIM...) & 3.1 & 6.1 & 3.4 & 4.1 & 3.2 \\
\hline Organisation privée internationale & & & & & \\
\hline CICR & 81.9 & 87.6 & 91.2 & 83.2 & 81.0 \\
\hline
\end{tabular}

Source: "Rapport du Conseil fédéral sur les relations entre la Suisse et l'Organisation des Nations Unies

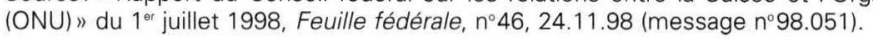

L'aide alimentaire s'est élevée à 32,3 millions de francs en 1997. La quasi-totalité de cette aide alimentaire a été acheminée par les ONG suisses (pour un mon- 
tant de 16,6 millions de francs) et par le PAM. L'aide alimentaire est constituée principalement par du lait en poudre, du blé et de la farine, du maïs et du riz.

Le tableau $\mathrm{n}^{\circ} 22$ montre les principaux pays ou régions bénéficiaires de l'aide humanitaire versée par la Suisse de 1995 à 1997. Cette aide s'est concentrée sur quatre régions: l'ex-Yougoslavie, la région des Grands Lacs (Rwanda et Burundi), la Corne de l'Afrique et les républiques du Caucase. Jamais la Confédération n'était intervenue autant que dans l'ex-Yougoslavie ${ }^{7}$. De 1991 à 1997, la Suisse a versé des contributions de plus de 1,5 milliard de dollars en lien avec les conséquences des conflits en ex-Yougoslavie; pendant la même période, elle a versé une aide humanitaire de 123 millions de dollars (par la DDC) et une contribution à la reconstruction de 52 millions de dollars (par la DDC et l'Office fédéral des réfugiés, dans le cadre de l'aide au retour de réfugiés bosniaques, pour les projets d'infrastructures). Les contributions en faveur de réformes politiques se sont élevées à 35 millions de dollars (promotion de la paix, soutien au processus électoral, bérets jaunes, aide aux médias indépendants). Seule une partie des contributions ci-dessus est incluse dans l'APD de la Suisse. L'assistance individuelle à la réintégration de réfugiés s'est élevée à 16 millions de dollars (Office fédéral des réfugiés). Les projets soutenus et financés par les ONG se sont élevés à 67 millions de dollars. A cela s'ajoutent des dépenses en Suisse de plus de 1,2 milliard de dollars pour l'accueil des réfugiés en Suisse (somme non incluse dans l'APD).

Tableau n²2: Aide humanitaire de la Confédération (montants cumulés de 1995 à 1997)

\begin{tabular}{|c|c|c|}
\hline & $\begin{array}{l}\text { Pays ou régions bénéficiaires } \\
\text { de l'aide humanitaire }\end{array}$ & En millions de francs \\
\hline 1. & Etats de I'ex-Yougoslavie & 60.8 \\
\hline 2. & Rwanda/région des Grands Lacs & 37.8 \\
\hline 3. & Angola & 21.9 \\
\hline 4. & Républiques du Caucase* & 17.7 \\
\hline 5. & Corée du Nord & 13.4 \\
\hline 6. & Ethiopie & 13.2 \\
\hline 7. & Russie & 11.0 \\
\hline 8. & Soudan & 10.6 \\
\hline 9. & Afghanistan & 8.8 \\
\hline 10. & Congo (ex-Zaïre) & 8.2 \\
\hline 11. & Somalie & 7.7 \\
\hline 12. & Erythrée & 7.6 \\
\hline 13. & Libéria & 7.5 \\
\hline 14. & Mozambique & 6.8 \\
\hline \multirow[t]{6}{*}{15.} & Irak & 6.5 \\
\hline & 15 pays/régions ci-dessus & 239.5 \\
\hline & Autres pays & 108.9 \\
\hline & Non ventilé géographiquement & 216.6 \\
\hline & Aide humanitaire multilatérale & 132.3 \\
\hline & Total cumulé de l'aide humanitaire & 697.3 \\
\hline
\end{tabular}

Source: DDC/OFAEE, Coopération internationale de la Suisse. Rapport annuel 1997 (brochure Aide humanitaire), Berne, 1998.

* Géorgie, Arménie et Azerbaïjan.

7. Voir la brochure de la DDC Swiss Commitment in Former Yugoslavia, August 1998, qui présente une description de l'engagement de la Suisse dans les Etats de l'ex-Yougoslavie. 


\subsection{AIDE MULTILATÉRALE ET COOPÉRATION INTERNATIONALE}

\section{$\square$ Rapport sur les relations entre la Suisse et l'ONU}

En réponse au postulat du conseiller national Andreas Gross (cosigné par 84 députés), le Conseil fédéral a présenté en juillet 1998 son rapport sur les relations entre la Suisse et l'Organisation des Nations Unies (ONU) $)^{8}$. Le rapport résume les buts et la structure de l'ONU ainsi que l'évolution des liens que la Suisse entretient avec l'organisation.

La Suisse accorde une grande importance à la concertation multilatérale. Les objectifs du Conseil fédéral exprimés dans son rapport de 1993 sur la politique extérieure de la Suisse dans les années 90 rejoignent ceux des Nations Unies. Les partisans de l'adhésion de la Suisse à l'ONU estiment que la situation internationale a complètement changé depuis 1986 lorsque le peuple suisse avait refusé cette adhésion. La récolte de signatures pour une nouvelle initiative en faveur de l'adhésion de la Suisse à l'ONU a débuté en septembre 1998 (100'000 signatures sont nécessaire d'ici mars 2000). Cette initiative est lancée par un groupe de personnalités politiques de divers partis ainsi qu'une trentaine d'ONG. Le Conseil fédéral a en outre annoncé en décembre 1998 qu'il allait proposer l'adhésion au plus tard en 2003.

\section{Importance de la concertation multilatérale}

Pour le Conseil fédéral, la fin de la guerre froide, la mondialisation, l'interdépendance entre pays, l'existence de problèmes complexes de portée globale (environnement, maintien de la paix, migrations par exemple) sont des facteurs qui rendent la coopération multilatérale plus importante encore qu' autrefois. Les débats internationaux naguère dominés par les conflits idéologiques Est-Ouest et Nord-Sud se sont orientés ces dernières années vers une appréhension plus complète des problèmes, avec notamment une série de grandes conférences mondiales pour examiner des thèmes de portée globale: droits de l'enfant en 1990, environnement et développement à Rio en 1992, droits de l'homme à Vienne en 1993, population au Caire en 1994, Conférence mondiale sur les femmes à Pékin et Sommet pour le développement social à Copenhague en 1995, Habitat II à Istanbul et Sommet mondial pour l'alimentation à Rome en 1996. La Suisse a participé à toutes ces conférences mondiales, avec des délégations composées de représentants de l'administration fédérale, mais aussi des acteurs de la société civile (en intégrant ces acteurs aux préparatifs, aux travaux et aux suivis des conférences). Bien que les programmes d'action adoptés lors de ces conférences soient dépourvus de tout caractère contraignant et qu'ils ne soient pas à la hauteur des espérances initiales, ils représentent des textes de référence. Le rapport du Conseil fédéral relève que le bilan souvent mitigé est dû moins au contenu des textes eux-mêmes qu'au manque de volonté politique de les mettre en œuvre de manière rigoureuse dans les Etats membres. Certaines conférences ont cependant débouché sur l'adoption d'accords internationaux contraignants (conventions sur

8. «Rapport du Conseil fédéral sur les relations entre la Suisse et l’Organisation des Nations Unies» du 1" juillet 1998, Feuille fédérale, $\mathrm{n}^{\circ} 46,24.11 .98$ (message $\mathrm{n}^{\circ} 98.051$ ).

9. Le rapport du Conseil fédéral énumérait cinq priorités: maintien et promotion de la sécurité et de la paix; engagement en faveur des droits de l'homme, de la démocratie et des principes de l'Etat de droit; accroissement de la prospérité commune; promotion de la cohésion sociale; préservation du milieu naturel. 
les changements climatiques et sur la diversité biologique), sur de nouveaux instruments internationaux ou de nouvelles structures (poste de haut-commissaire des Nations Unies pour les droits de l'homme par exemple).

\section{$\square$ Participation de la Suisse aux institutions multilatérales}

Bien qu'elle ne soit pas membre de l'ONU, la Suisse participe financièrement à de nombreux programmes multilatéraux et est membre à part entière de toutes les agences spécialisées. Le schéma suivant met en évidence les organisations du système des Nations Unies dont la Suisse est membre (en gras/italique) ou que la Suisse soutient financièrement (avec un double astérisque). La Suisse s'associe à la plupart des activités opérationnelles des Nations Unies, dans les domaines du maintien de la paix, des droits de l'homme, de la coopération au développement, des actions humanitaires et de l'environnement. Un tiers de toute l'aide humanitaire de la Confédération passe par des organisations multilatérales actives dans ce domaine, et un autre tiers de l'aide humanitaire passe par le CICR. L'aide multilatérale représente aussi un tiers de toute la coopération au développement à plus long terme.

Le statut d'observateur auprès de l'ONU se révèle selon l'avis du Conseil fédéral de plus en plus insatisfaisant. La Suisse fait partie des pays qui apportent le plus de contributions financières tout en ne pouvant pas être représentée dans certains organes de décision. Lorsque par exemple la Suisse veut prendre la parole à l'Assemblée générale des Nations Unies, elle doit accomplir une procédure complexe pour vérifier qu'aucun Etat membre ne s'y oppose. Le poids politique des Etats ou mouvements ayant le statut d'observateur est faible (Suisse, Saint-Siège, les îles du Pacifique Tonga et Nauru, le mouvement de l'OLP). Les autres observateurs sont des organisations privées internationales ou des ONG (dont le CICR et la Fédération internationale des sociétés de la Croix-Rouge et du Croissant Rouge). La Suisse peut cependant être élue comme membre à part entière dans plusieurs commissions (Commission pour le développement durable par exemple) ou comités des Nations Unies (tels qu'ONUSIDA), ainsi que dans certains conseils d'administration (PNUD, PNUE, UNICEF, FNUAP, HCR)

En prenant pour base de calcul l'année 1998, une adhésion de la Suisse à l'ONU ne représenterait une charge supplémentaire totale que de 35,7 millions de francs environ (13,2 millions de francs de charges supplémentaires au budget ordinaire de l'ONU et 22,5 millions de francs de charges pour les contributions obligatoires aux opérations de maintien de la paix). Ce sont des montants supplémentaires à payer car la Suisse finance déjà une partie du budget ordinaire de l'ONU (4,9 millions de francs en 1996) et verse déjà une contribution spécifique (volontaire) aux opérations de maintien de la paix (13,4 millions de francs en 1997). La Suisse verse donc actuellement au budget ordinaire de l'ONU une somme correspondant à $30 \%$ de la contribution qu'elle aurait à verser si elle était membre à part entière. 


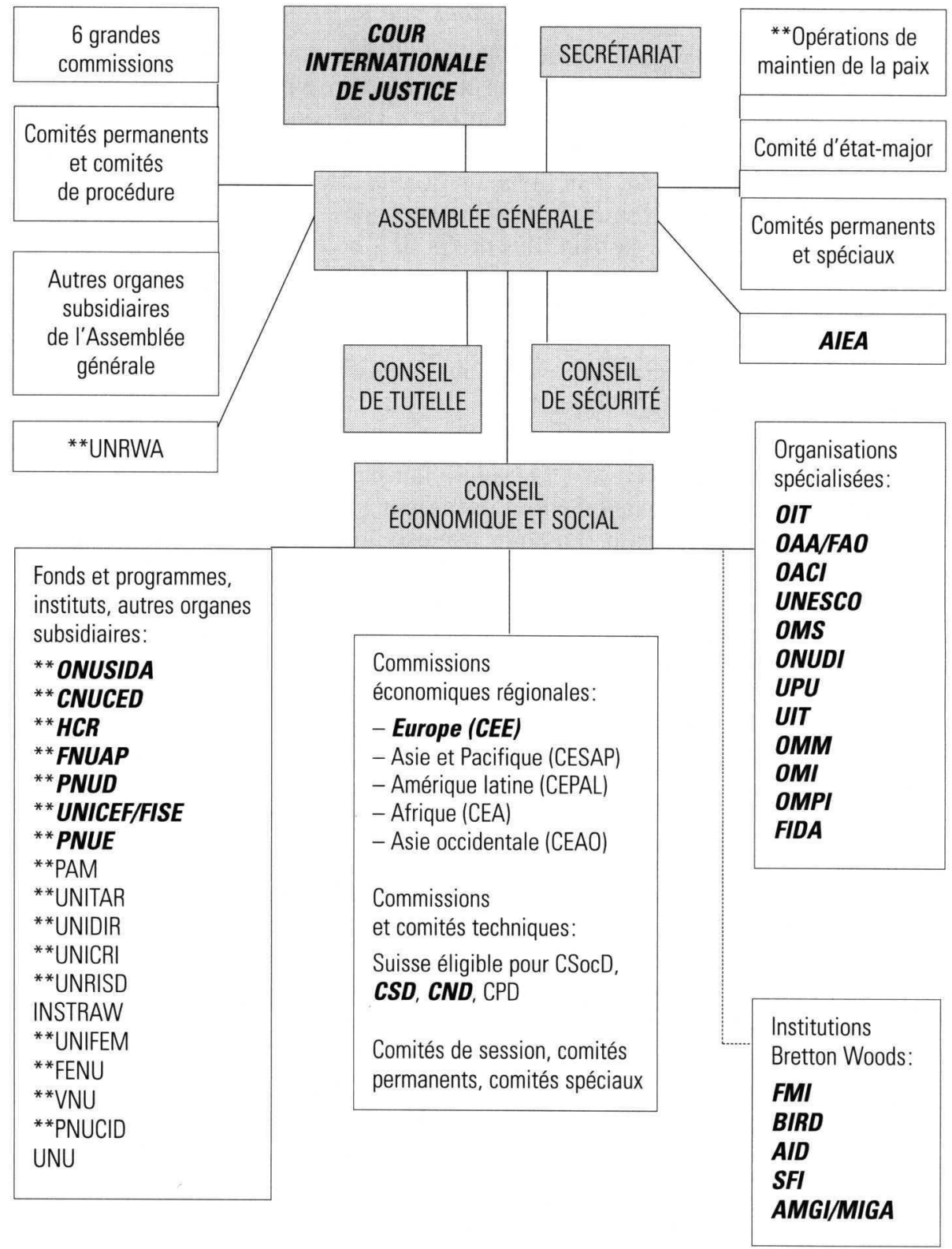

Source: «Rapport du Conseil fédéral sur les relations entre la Suisse et I'Organisation des Nations Unies » du

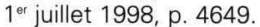

En caractère gras/italique: la Suisse est membre ou partie contractante (pour les organes subsidiaires: membre de l'organe directeur).

*Pour les définitions en toutes lettres des sigles contenus dans ce schéma, se reporter à la liste des abréviations en fin d'ouvrage.

**La Suisse verse des contributions volontaires. 


\section{$\square$ Contributions de la Suisse aux organisations multilatérales}

L'aide multilatérale de la Suisse s'est élevée à 376 millions de francs en 1997. La part de l'aide multilatérale dans l'APD totale est en constante progression ces dernières années (en passant de 23\% de l'APD en 1993 à 31\% en 1997). En 1997, la Suisse a versé 139 millions de francs au Groupe de la Banque mondiale, 54 millions de francs aux banques régionales de développement, 71 millions de francs à des organisations internationales actives dans l'aide humanitaire (sans le CICR) et 121 millions de francs aux autres organismes des Nations Unies. Le tableau suivant montre l'évolution des contributions au système de l'ONU depuis 1993.

Tableau n²3: Contributions de la Suisse au système des Nations Unies (1993-1997) en millions de francs (montants compris dans l'aide publique au dév.)

\begin{tabular}{|c|c|c|c|c|c|}
\hline & 1993 & 1994 & 1995 & 1996 & 1997 \\
\hline Groupe de la Banque mondiale & 133.0 & 151.3 & 146.9 & 183.0 & 138.9 \\
\hline Banques régionales de développement & 51.8 & 63.6 & 53.1 & 50.1 & 54.0 \\
\hline $\begin{array}{l}\text { Organisations des Nations Unies (NU) } \\
\text { actives dans la coopération au dév. }\end{array}$ & 148.6 & 149.7 & 135.3 & 131.1 & 120.6 \\
\hline $\begin{array}{l}\text { Organisations des Nations Unies } \\
\text { actives dans I'aide humanitaire }\end{array}$ & 92.3 & 85.0 & 73.6 & 80.9 & 71.3 \\
\hline Total des contributions aux NU & 425.7 & 449.6 & 408.9 & 445.1 & 384.8 \\
\hline Total de l'APD & 1221.6 & 1317.4 & 1269.1 & 1266.1 & 1217.0 \\
\hline $\begin{array}{l}\text { Dont APD comptabilisée } \\
\text { sous aide multilatérale }\end{array}$ & 2840 & 3323 & 3480 & 3727 & 376.0 \\
\hline Aide multilatérale en \% de l'APD & 23.25 & 25.22 & 27.42 & 29.44 & 30.90 \\
\hline
\end{tabular}

Source: "Rapport du Conseil fédéral sur les relations entre la Suisse et l'Organisation des Nations Unies » du $1^{\text {er }}$ juillet 1998, annexes 8 et 9.

\section{Contributions financières de la Suisse} et part comprise dans l'aide publique au développement (APD)

Il faut relever que l'aide de la Suisse transitant par les organisations internationales (soit 470 millions de francs sans le CICR) est plus importante que le chiffre publié de l'aide multilatérale (376 millions de francs en 1997), cela pour les trois raisons suivantes:

- Les contributions pour financer des projets particuliers de certaines organisations internationales sont comptabilisées dans l'aide bilatérale lorsque le pays donateur conserve un contrôle direct sur le choix des projets et l'emploi des fonds (aide associée et cofinancements). L'aide est multilatérale lorsque les contributions des différents pays donateurs sont fondues en une seule masse et les projets choisis par les instances de décision de l'organisation multilatérale. La coopération internationale totale comprend donc l'aide multilatérale proprement dite (contributions générales à une organisation) et l'aide dite «multi-bilatérale», avec les contributions affectées à des programmes spécifiques choisis par la Suisse.

- Les contributions aux organisations privées internationales comme le CICR sont comptabilisées dans l'aide bilatérale (versements non compris dans le tableau $\mathrm{n}^{\circ} 23$ ).

- Les contributions pouvant être comptabilisées dans l'APD ne représentent qu'une partie des contributions de la Suisse aux Nations Unies et autres orga- 
nisations internationales. Selon les directives de l'OCDE, une contribution ne peut être comptabilisée sous l'APD multilatérale que si l'institution bénéficiaire consacre tout ou partie de ses activités au développement et aux pays bénéficiaires de l'aide. Les contributions aux organisations multilatérales qui ne consacrent qu'une partie de leurs activités au développement ne sont prises en compte dans l'APD qu'à concurrence de la partie de leur travail correspondant à leurs activités en faveur du développement. Le CAD révise périodiquement la liste des organisations dont les contributions peuvent être (entièrement ou en partie) comptabilisées dans l'APD. Par exemple, 84\% des contributions au Fonds pour l'environnement mondial peuvent ainsi être comptés dans l'APD depuis 1997. Les trois quarts des contributions ordinaires à l'OMS et la totalité des contributions aux programmes spéciaux de l'OMS peuvent être comptabilisés dans l'APD. Les contributions ordinaires aux organisations suivantes ne peuvent être que partiellement comptabilisées dans l'APD: OMPI, FAO, OIT, OMM, UPU, UIT, UNESCO.

Si l'on tient compte des éléments ci-dessus, près de la moitié de l'APD fournie par la Suisse transite par une organisation internationale.

\section{$\square$ Importance économique de la Genève internationale}

Les retombées économiques de la présence des organisations internationales à Genève sont difficiles à évaluer. Le rapport du Conseil fédéral donne quelques estimations dans ce domaine ${ }^{10}$. Genève accueille plus d'une vingtaine d'organisations internationales intergouvernementales et plus de 120 organisations non gouvernementales à caractère international. Cent quarante Etats sont représentés à Genève par une mission permanente. La présence des organisations internationales entraîne la présence permanente de plus de 30'000 personnes à Genève (et dans les environs) ${ }^{11}$, ainsi que le séjour d'environ 90'000 personnes par année à l'occasion des diverses conférences. A Genève, plus d'un poste de travail sur dix relève du secteur international et les organisations sont à l'origine d'un volume de commandes en biens et services pour un montant estimé à 1,5 milliard de francs environ. La Suisse figure parmi les pays ayant obtenu les plus grosses parts du volume de commandes de l'ONU. L'ONU et l'OMS achètent par exemple plus de la moitié de la production mondiale de vaccins, ce qui profite aussi à l'industrie suisse des produits pharmaceutiques. L'apport économique annuel total de la Genève internationale est évalué dans le rapport à 2,5 à 3 milliards de francs; 55’000 Suisses travaillent dans le système des Nations Unies, dont 31'000 au sein des organisations spécialisées. La concurrence pour accueillir des conférences internationales ou des secrétariats d'organisations s'est accrue car d'autres villes affichent leur volonté d'accueil. Genève a obtenu ces dernières années le siège de l'OMC; c'est par contre Bonn qui s'est vu attribuer le Secrétariat de la Convention-cadre sur les changements climatiques et celui de la convention sur la désertification. Le siège de la Convention sur la biodiversité a été établi à Montréal, celui de l'Organisation pour l'interdiction des armes chimiques à La Haye.

CaSTM 1998, dans le chapitre 5 de la «Revue», voir le passage sur l'ONU (pp. 215-221).

10. Les chiffres de ce paragraphe sont tirés du «Rapport du Conseil fédéral sur les relations entre la Suisse et l'Organisation des Nations Unies» du $1^{\text {er juillet } 1998 .}$

11. Fonctionnaires internationaux et diplomates, avec leur famille et leur personnel domestique. 


\subsection{BANQUES RÉGIONALES DE DÉVELOPPEMENT (AFRICAINE, ASIATIQUE ET INTERAMÉRICAINE)}

$\square$ Participation de la Suisse aux travaux des banques régionales de développement

La Suisse est membre de la Banque asiatique depuis 1967 et membre des deux autres banques régionales depuis leur ouverture à des pays extérieurs à la région (1976 pour la BID et 1982 pour la BAD). L'arrêté fédéral concernant la participation de la Suisse à l'augmentation du capital des Banques de développement entré en vigueur le 19 décembre 1995 constitue la base légale des engagements de la Suisse (voir l'Annuaire 1996, pp. 155-160, pour la teneur du message relatif à cette augmentation de capital). Ce crédit de programme de 800 millions de francs a été ouvert pour la participation de la Suisse à l'augmentation du capital des banques de développement interaméricaine, asiatique et africaine, ainsi que de la Société interaméricaine d'investissement et de l'Agence multilatérale de garantie des investissements (AMGI). Seuls 45 millions de francs seront effectivement libérés sur les 800 millions, le reste du montant constituant un capital de garantie. Les contributions de la Suisse aux banques régionales de développement et à leurs fonds spéciaux se sont élevées à 54 millions de francs en 1997.

\section{Fonctionnement général des banques de développement}

Les banques régionales sont des institutions financières multilatérales dont le but est de soutenir des projets et programmes favorisant le développement, en octroyant des prêts et une assistance technique. Les ressources financières des banques régionales de développement sont composées du capital-actions de base, des emprunts effectués sur le marché international des capitaux, des contributions des pays donateurs aux fonds de développement ainsi que des revenus de placements à court terme. Les banques régionales de développement fournissent deux types de crédits, l'un aux conditions du marché pour les pays les mieux lotis de la région, l'autre à des taux favorables pour les pays les plus démunis, par le biais des fonds de développement de chacune des banques (fonds concessionnels). Grâce à leur capital largement réparti et aux garanties des pays membres, les banques régionales de développement peuvent emprunter sur les marchés internationaux les fonds supplémentaires afin de financer des investissements prioritaires en matière de développement. Les membres des banques régionales de développement sont divisés en deux grands groupes: les pays du continent concerné (pays régionaux) et certains pays d'autres régions (pays industrialisés surtout) qui détiennent une partie du capital de base et financent les fonds régionaux pour les pays les plus pauvres. Cela explique l'intérêt des pays industrialisés non seulement à financer ces institutions, mais aussi à prendre part aux décisions et à suivre la gestion des banques et les politiques d'affectation des crédits.

Les négociations périodiques relatives aux augmentations de capital des banques et aux reconstitutions de leur fonds de développement sont des moments cruciaux où les priorités de travail pour les années à venir sont fixées. Les trois banques régionales sont toutes confrontées depuis quelques années à la nécessité d'améliorer l'efficacité de leurs activités opérationnelles (programmes et projets) et d'entreprendre des réformes institutionnelles. Les banques régionales doivent accorder une attention plus grande à la gouvernance dans les Etats du Sud: amélioration de l'efficacité de la gestion du secteur public, promotion de l'engagement du secteur public dans le secteur social tout en réduisant les dépenses non productives telles que les dépenses militaires, lutte contre la corruption.

Les banques régionales de développement sont dirigées par un conseil des gouverneurs (dans lequel chaque pays membre siège avec un gouverneur et un suppléant) et par un conseil d'administration (avec des administrateurs représentant 
un groupe de pays). Les gouverneurs suisses sont des représentants de l'OFAEE et les gouverneurs suppléants viennent de la DDC. La Suisse forme avec d'autres pays (extérieurs aux régions concernées) un groupe de vote qui désigne à tour de rôle les représentants au conseil d'administration (soit un administrateur, un administrateur suppléant, un conseiller et un assistant de l'administrateur). Le tableau suivant résume quelques caractéristiques des trois banques régionales.

Tableau n²4: Données sur trois banques régionales de développement

\begin{tabular}{|c|c|c|c|}
\hline & $\begin{array}{l}\text { Banque africaine } \\
\text { de développement }\end{array}$ & $\begin{array}{l}\text { Banque asiatique } \\
\text { de développement }\end{array}$ & $\begin{array}{c}\text { Banque interaméricaine } \\
\text { de développement }\end{array}$ \\
\hline Nombre de pays membres & 77 pays & 57 pays & 46 pays \\
\hline Pays régionaux (\% droits de vote) & $53(65.8 \%$ des voix) & $41(65 \%$ des voix env.) & $28(87.4 \%$ des voix $)$ \\
\hline Pays non régionaux & & & (USA: $31.8 \%$ des voix) \\
\hline (\% droits de vote) & $24(34.2 \%$ des voix) & 16 (35\% des voix env.) & $18(12.6 \%)$ \\
\hline Capital de la banque & $29 \mathrm{mia} . \$$ & $80 \mathrm{mia} . \$$ & $80 \mathrm{mia} . \$$ \\
\hline Dernière augmentation du capital & 1998 & 1994 & 1994 \\
\hline $\begin{array}{l}\text { Volume des prêts approuvés en } 19 \\
\text { (en millions de dollars) }\end{array}$ & 1776.6 & 9414.0 & 6017.3 \\
\hline sur capital ordinaire & 771.1 & 7794.4 & 5649.0 \\
\hline sur Fonds concessionnel & 1005.5 & 1619.6 & 283.4 \\
\hline Fonds spéciaux & & & 84.9 \\
\hline $\begin{array}{l}\text { Dernière reconstitution du Fonds } \\
\text { concessionnel de développement }\end{array}$ & 1996 & 1997 & 1994 \\
\hline Montant et période & 3 mia. $\$$ de 96 à 98 & 6.3 mia. $\$$ de 97 à 00 & $\begin{array}{c}2 \text { mia. } \$ \text { de } 94 \text { à } 97 \\
1.2 \text { mia. } \$ \text { pour } 98 \text { et } 99\end{array}$ \\
\hline $\begin{array}{l}\text { Part de la Suisse au capital de la ba } \\
\text { (et donc\% droits de vote) }\end{array}$ & anque $1.27 \%$ & $0.84 \%$ & $0.47 \%$ \\
\hline $\begin{array}{l}\text { Représentation suisse au sein du } \\
\text { conseil d'administration }\end{array}$ & $\begin{array}{c}\text { Une assistante de } \\
\text { I'administratrice de } \\
97 \text { à juin } 99\end{array}$ & $\begin{array}{l}\text { Un administrateur } \\
\text { suppléant de } \\
96 \text { à l'été } 98\end{array}$ & $\begin{array}{l}\text { Un administrateur } \\
\text { suppléant de } \\
96 \text { à l'été } 98\end{array}$ \\
\hline
\end{tabular}

Sources: rapports annuels des banques de développement et OFAEE.

\section{$\square$ Banque africaine de développement}

Le conseil des gouverneurs de la Banque africaine de développement (BAD) est composé de représentants des 53 pays membres de la région et de 24 pays non régionaux. Le gouverneur de la Suisse auprès de la BAD (et au sein du conseil des gouverneurs du Fonds africain pour le développement - FAD) est l'ambassadeur Nicolas Imboden, de l'OFAEE, Henri Philippe Cart, de la DDC, étant le gouverneur suppléant. Le conseil d'administration de la banque est composé de 18 membres, 12 représentants de pays de la région et 6 représentants de pays extérieurs à la région. Le groupe de vote auprès du conseil d'administration se compose de la Suède, du Danemark, de la Finlande, de la Norvège, de la Suisse et de l'Inde. Selon le système de rotation, c'est la Finlande qui occupe le poste d'administrateur pour ce groupe de vote (jusqu'en 2001) (une Suissesse est assistante de l'administratrice jusqu'en été 1999).

\section{Réformes dans le fonctionnement de la banque}

La Suisse suit attentivement les progrès de la mise en œuvre des réformes institutionnelles et financières de la banque entamées dès $1995^{12}$. Ces réformes englo-

12. Voir par exemple la partie consacrée aux réformes dans les banques régionales de développement publiée dans le «Rapport sur la politique économique extérieure $97 / 1+2 »$ du 19 janvier 1998 , Feuille fédérale, n $9,10.3 .98$, pp. 759761 (message $\left.\mathrm{n}^{\circ} 97.090\right)$. 
bent notamment les domaines suivants: amélioration de la qualité des opérations de la banque et des projets soutenus (avec une meilleure évaluation des résultats et l'établissement de stratégies par pays pour orienter le programme de prêt sur trois ans, une augmentation des performances des agences d'exécution et une amélioration de la gestion financière), meilleure formation du personnel (et recrutement de personnel plus qualifié), réformes dans le fonctionnement de la banque.

\section{Activités opérationnelles}

Les prêts du Groupe de la Banque africaine se sont élevés à 1,8 milliard de dollars en 1997 (contre 800 millions en 1996), dont 771 millions de dollars de prêts de la BAD et un peu plus d'un milliard de dollars de dons et prêts du Fonds africain pour le développement (guichet concessionnel pour les pays les plus pauvres). Les activités du FAD étaient paralysées en 1994 et 1995 (aucun prêt avant la reconstitution du FAD, décidée en 1996), les prêts sur le FAD s'élevant ensuite à 286 millions de dollars en 1996 (pour comparaison, les prêts du FAD se situaient entre 900 et 1160 millions dans les années 1989 à 1993). Cette paralysie des activités du FAD était notamment due aux difficiles négociations en vue de la reconstitution du FAD adoptée en 1996.

Les prêts de la banque se sont dirigés surtout vers les pays de l'Afrique du Nord (59\% du total des prêts) et vers l'Afrique australe (34\%). Les pays de l'Afrique de l'Ouest (38\%) et de l'Afrique de l'Est (30\%) sont les principaux bénéficiaires du FAD. Les secteurs concernés par les prêts de la banque sont essentiellement l'industrie ( $40 \%$ des prêts), les services publics (16\%) et les transports (12\%). Les secteurs agricoles et sociaux représentent $39 \%$ des prêts du FAD, ce qui constitue une part encore bien inférieure à l'objectif de $60 \%$ qui avait été fixé lors des négociations de la $7^{\mathrm{e}}$ reconstitution du FAD. Plusieurs pays membres de la BAD extérieurs à la région (mais bailleurs de fonds importants du FAD) souhaiteraient que la banque concentre ses activités sur la lutte contre la pauvreté.

\section{Augmentation du capital de la banque}

La $34^{\mathrm{e}}$ assemblée annuelle de la Banque africaine de développement (BAD) et la $25^{\mathrm{e}}$ assemblée du Fonds africain pour le développement se sont déroulées fin mai 1998 à Abidjan. Après deux ans de négociations laborieuses, les gouverneurs de la BAD ont conclu un accord portant sur une augmentation de $35 \%$ du capital de la banque, qui passe ainsi de 21 à 29 milliards de dollars. La part des pays extérieurs à la région au capital total est plus grande qu'auparavant (passant d'un tiers à $40 \%$ ). L'augmentation du capital permet à la banque de renforcer son assise financière (en obtenant par exemple des conditions d'emprunts plus avantageuses sur les marchés financiers internationaux). Le point le plus controversé des négociations a été la question de la répartition des voix entre pays régionaux et pays extérieurs à la région lors des votes. Les pays non régionaux souhaitaient augmenter leur influence dans les prises de décision, alors que certains grands pays régionaux craignaient une perte trop grande du caractère africain de la banque. Le compromis trouvé sur les règles de vote indique que les décisions doivent être prises généralement à la majorité des deux tiers des voix, mais qu'une majorité de $70 \%$ peut être appliquée exceptionnellement lorsqu'un administrateur en fera expressément la demande en fonction de l'importance de la décision pour les pays qu'il représente. Plusieurs pays non régionaux ont exprimé leur insatisfaction quant au compromis trouvé, les règles de vote étant 
trop basées sur une logique de confrontation et de minorité de blocage, alors qu'il faudrait développer un partenariat entre pays et un consensus sur certaines questions. Suite à l'accord ci-dessus, les pays non régionaux ont entamé la négociation visant à reconstituer le Fonds africain pour le développement ( $8^{\mathrm{e}}$ reconstitution du FAD).

\section{Réduction de l'endettement}

La Suisse a signé en décembre 1998 un accord avec le Fonds africain pour le développement pour une contribution de 8 millions de francs au Mécanisme supplémentaire de financement du FAD. Ce mécanisme a été créé en 1997 pour aider les pays membres régionaux à faible revenu à payer les arriérés de paiement d'intérêts sur les crédits de la BAD. Cette mesure fait partie des programmes visant à réduire la dette extérieure des pays les plus pauvres (avec l'initiative PPTE par exemple). Ce mécanisme du FAD a profité à 12 pays en 1998, pour un montant total de 59 millions de dollars.

\section{$\square$ Banque asiatique de développement}

La $31^{\mathrm{e}}$ assemblée de la Banque asiatique de développement (BAsD) s'est tenue à Genève du 29 avril au $1^{\text {er }}$ mai 1998. C'était la première fois que la Suisse accueillait l'assemblée annuelle d'une banque régionale de développement. La délégation suisse était composée des conseillers fédéraux Flavio Cotti et Pascal Couchepin, de l'ambassadeur Nicolas Imboden (OFAEE), de deux autres représentants de l'OFAEE et d'un représentant de la DDC. Nicolas Imboden a présidé le conseil des gouverneurs de l'été 1997 à l'été 1998. Pendant l'assemblée, plusieurs séminaires ont été organisés conjointement par la Banque asiatique, des instituts universitaires, des organisations internationales et des banques (par exemple sur la crise financière asiatique, le développement social, la santé, les télécommunications et le secteur urbain). Le Tadjikistan est devenu en 1998 le $57^{\circledR}$ pays membre de la BAsD. La Suisse a facilité l'adhésion de ce pays en finançant sa part au capital de la banque (le Tadjikistan fait partie du groupe de vote de la Suisse auprès du FMI et de la Banque mondiale).

Dans son allocution d'ouverture de l'assemblée annuelle, le conseiller fédéral Flavio Cotti a relevé l'importance de la bonne gestion des affaires publiques pour un développement durable profitant à l'ensemble de la société. «Les fondements essentiels d'un tel processus sont l'Etat de droit, une administration publique transparente, la justice, une répartition aussi équitable que possible des revenus, des rapports équilibrés villes-campagnes, des institutions à travers lesquelles les citoyens et les citoyennes peuvent s'exprimer et des moyens d'information libres et responsables. ${ }^{13}$

En marge de l'assemblée, la Suisse a annoncé une contribution de 2 millions de dollars pour la création d'un fonds fiduciaire en faveur de la promotion du secteur privé. Le conseiller fédéral Pascal Couchepin et le directeur de la DDC Walter Fust ont aussi eu des entretiens bilatéraux avec une douzaine de délégations de pays asiatiques.

Interventions de la BAsD suite à la crise asiatique

Les discussions de l'assemblée annuelle ont été dominées par la crise asiatique. Pour contrecarrer cette crise et financer des opérations de sauvetage, la Banque

13. Opening Address by Flavio Cotti, 29.4.98. 
asiatique s'est engagée massivement aux côtés des institutions de Bretton Woods. Le montant des engagements de la BAsD a ainsi quasiment doublé, en passant de 5,5 milliards de dollars en 1996 à 9,4 milliards de dollars en 1997, dont 7,8 milliards de dollars de prêts sur le capital ordinaire et 1,6 milliard de prêts du Fonds concessionnel asiatique de développement (FAsD). Les engagements financiers se sont orientés surtout sur le soutien des réformes dans les secteurs financier et bancaire des pays en crise (la réforme des marchés financiers a absorbé la moitié des prêts de la banque, FAsD compris). Les principaux pays bénéficiaires des crédits sur le capital ordinaire ont été en 1997 la Corée du Sud (avec un crédit record de 4 milliards de dollars pour le secteur financier ${ }^{14}$, soit le plus gros programme accordé depuis la création de la banque), l'Indonésie (1,1 milliard de crédits), la Chine, l'Inde et la Thaillande (550 millions). Les pays en crise dont les montants sont mentionnés ci-dessus ont absorbé à eux seuls plus de $72 \%$ des prêts sur le capital ordinaire. Les principaux pays bénéficiaires des ressources du FAsD ont été le Bangladesh (420 millions de dollars), le Vietnam (360 millions de dollars) et le Pakistan (251 millions).

Si beaucoup de pays ont relevé lors de l'assemblée annuelle que la réponse de la Banque asiatique pour enrayer la crise était rapide et bienvenue, certains pays ont toutefois exprimé leur inquiétude quant à l'ampleur des ressources utilisées pour cette intervention d'urgence par rapport à la mission première de la banque, qui devrait être un appui pour le développement à long terme. Plusieurs pays ont notamment mis l'accent sur l'importance du mandat de la banque dans la lutte contre la pauvreté. Les prêts massifs opérés en 1997 ont été accompagnés d'une augmentation des réserves de la banque et donc d'une diminution des ressources disponibles pour des activités d'assistance technique ou pour financer le Fonds asiatique de développement (le FAsD est financé conjointement par les pays donateurs et par les contributions du revenu net du capital de la banque).

\section{$\square$ Banque interaméricaine de développement}

La Banque interaméricaine de développement (BID) participe au financement de programmes et projets des pays membres (tous les pays latino-américains sauf Cuba). Elle peut aussi accorder des prêts sans garantie gouvernementale au secteur privé de ces pays. La Société interaméricaine d'investissement (SII) fournit des prêts à des PME ou des intermédiaires financiers; 35 pays sont membres de la SII (contre 46 pays membres de la BID), dont 10 pays extérieurs à la région (dont la Suisse). L'assemblée annuelle du conseil des gouverneurs de la banque et de la SII s'est déroulée à Cartagena de Indias (Colombie) en mars 1998. L'assemblée annuelle 1999 des deux institutions aura lieu à Paris, du 15 au 17 mars.

\section{Activités opérationnelles}

Le montant total des prêts accordés par la banque a été de 6 milliards de dollars en 1997 (contre 6,7 milliards l'année précédente), dont 5,6 milliards de dollars sur le capital de la banque et 283 millions sur le Fonds concessionnel. Les principaux pays bénéficiaires des prêts opérés sur le capital ordinaire de la banque sont le Brésil (prêts s'élevant à 1,5 milliard de dollars en 1997), l'Argentine (1,2 milliard) - ces deux pays absorbant $48 \%$ du volume total des prêts -, l'Uru-

\footnotetext{
14. Le programme de réforme du secteur financier coréen comprend des mesures de capacity building en faveur du secteur bancaire, l'établissement d'infrastructures pour le marché financier et des mesures pour l'amélioration du contrôle des sociétés privées et des standards d'information.
} 
guay, le Panama, la Colombie et le Pérou. Les principaux pays bénéficiaires des prêts du Fonds concessionnel (Fund for Special Operations FSO) ont été le Nicaragua, Haïti, la Guyane et l'Equateur.

\section{Assemblée annuelle}

L'assemblée annuelle a permis d'examiner le projet de "vision de la banque» préconisant une stratégie de partenariat entre pays membres pour les années à venir et définissant les modalités d'une adaptation du mandat de la banque aux nouvelles réalités économiques de la région (nouveaux produits, développement du travail avec la société civile, appui au secteur privé). Les autres points principaux à l'ordre du jour étaient la question du financement de projets du secteur privé par la banque et le problème des ressources financières pour les pays les plus pauvres. Dans le cadre des discussions sur la vision d'avenir de la banque, des voix se sont élevées en faveur d'un assouplissement des règles d'attribution des crédits (volonté de certains pays régionaux surtout). Selon les priorités définies lors de la $8^{e}$ augmentation du capital de la banque en 1994 (BID-8), au moins $40 \%$ des prêts devraient soutenir le secteur social (pris dans un sens assez large ${ }^{15}$ ) et $35 \%$ devraient être octroyés aux pays les plus pauvres de la région ${ }^{16}$. En 1997, $43 \%$ des crédits concernaient le secteur social et les pays les plus pauvres représentaient $27 \%$ des crédits totaux de la banque. Certains pays souhaitaient aussi une augmentation du plafonnement des prêts directs de la BID au secteur privé (en relevant la part actuelle de 5\% au maximum du total des prêts de la banque à $10 \%$ ) ainsi qu'une augmentation de la part du projet pouvant être prise en charge par la banque. Les études se poursuivront car aucun consensus n'a été dégagé pour l'instant sur l'assouplissement de ces règles. La plupart des pays non régionaux (qui détiennent une partie du capital de la banque et qui apportent un financement au Fonds concessionnel) ont réaffirmé leur attachement aux objectifs fixés lors de l'augmentation du capital BID-8.

Suite aux engagements pris lors des négociations de BID-8, la banque a approuvé en 1997 de nouvelles lignes directrices dans certains domaines: classification des prêts visant la lutte contre la pauvreté, stratégies dans les secteurs de l'énergie et de l'éducation, soutien à l'initiative PPTE, procédure d'adjudication pour lutter contre la corruption, prêts sans garantie gouvernementale au secteur privé. La politique de soutien des mesures d'ajustement structurel est actuellement en révision, avec notamment le projet d'établir des prêts de soutien à l'ajustement institutionnel ${ }^{17}$. Ces prêts représenteraient une aide au budget pour couvrir certains coûts de l'ajustement (non pas par une aide à la balance des paiements, mais par des dépenses budgétaires de l'Etat). La question délicate dans ce domaine est d'assurer un financement pour des investissements et non pas pour couvrir des déficits budgétaires de fonctionnement).

Les discussions en séance plénière ont relevé que les performances économiques généralement favorables enregistrées en Amérique latine cachent d'importantes disparités entre pays et que ces résultats positifs n'ont pas été accompagnés des avancées attendues dans le domaine social. Les inégalités sociales et la pauvreté

15. Le domaine social comprend les secteurs suivants: santé et hygiène, développement urbain, éducation, investissements sociaux, environnement, microentreprise.

16. Pays les plus pauvres et petits pays = tous les pays d'Amérique latine, sauf l'Argentine, le Brésil, le Chili, la Colombie, le Mexique, le Pérou et le Venezuela.

17. IAL: Institutional Adjustment Lending. 
sont en progression malgré la forte croissance économique, la baisse de l'inflation et l'augmentation des flux de capitaux privés.

Reconstitution du Fonds concessionnel de la BID (FSO)

Les négociations relatives à la $9^{\mathrm{e}}$ reconstitution du FSO sont retardées en raison des divergences de vues entre les pays donateurs et pays emprunteurs quant au mode de financement (utiliser les ressources internes à la banque ou obtenir de nouvelles contributions des pays donateurs). Le conseil des gouverneurs a conclu un accord pour les années 1998 et 1999 en puisant encore dans les ressources du fonds (1,2 milliard de dollars). Cet accord permet de maintenir les prêts pour les pays les plus pauvres et de contribuer à l'initiative pour la réduction de la dette pour la Bolivie et la Guyane (initiative PPTE), mais la question des ressources concessionnelles dès l'an 2000 reste non résolue. Une augmentation du capital de la Société interaméricaine d'investissement n'a pour l'instant pas pu être conclue en raison de l'opposition des Etats-Unis, alors que la plupart des pays européens étaient favorables à une augmentation de ce capital.

[4] ASTM 1998, pp. 339-341 sur l'augmentation du capital de la BERD. 


\section{SOURCES}

\section{Evolution générale de l'aide publique au développement des pays du CAD}

OCDE, «L'aide et les apports de capitaux privés ont diminué en 1997», Communiqué de presse, Paris, OCDE, 18.6.98.

OCDE, Coopération pour le développement. Efforts et politiques des membres du Comité d'aide au développement (rapport 1997), Paris, OCDE, 1998.

Aide publique fournie par la Suisse

«Rapport du Conseil fédéral sur les relations entre la Suisse et l'Organisation des Nations Unies (ONU) établi en réponse au postulat déposé le 18 juin 1997 par le conseiller national Andreas Gross» du 1" juillet 1998, Feuille fédérale, $\mathrm{n}^{\circ} 46,24.11 .98$, pp. 4606-4672 (rapport n 98.051 ).

«Rapport sur la coopération internationale de la Suisse avec les pays du Sud et de l'Est 1986-1995, donnant suite au postulat n'96.3405 Zapfl du 17 septembre 1996» du 6 mai 1998.

«Rapport sur la politique économique extérieure 97/1+2» du 19 janvier 1998, Feuille fédérale, no $9,10.3 .98$, pp. 759-761 (message no 97.090).

DDC, Agenda politique, Berne, parution mensuelle.

DDC, Newsletter (informations en bref), 6 numéros par année.

DDC, Politique sectorielle DDC dans le domaine financier, série Politiques sectorielles de la DDC, Berne, avril 1998, $46 \mathrm{p}$.

DDC, Promotion des droits de l'homme et coopération au développement, DDC, Berne, 1997, 24 p.

DDC, Service de Presse, Berne, parution 4 fois par an.

DDC/OFAEE, Si les pauvres sont pauvres, c'est de leur faute. Questions et réponses au sujet de la coopération internationale de la Suisse (argumentaire sur la coopération au développement), DDC/OFAEE, Berne, 1999, 36 p.

DDC/OFAEE, Coopération internationale de la Suisse. Rapport annuel 1997 (3 brochures sur Aide humanitaire, Coopération au développement et Coopération avec l'Europe de l'Est), DDC/OFAEE, Berne, 1998.

DFAE, La Suisse et le monde (revue), parution 4 fois par année (commande: tél. 031/322.31.53).

\section{SITES INTERNET ET COMMANDE DE PUBLICATIONS}

Corps suisse d'aide en cas de catastrophe: http://www.skh.ch

DDC: http://www.sdc-gov.ch

DFAE: http://www.dfae.admin.ch Ce site contient tout un chapitre sur les organisations internationales: www.dfae.admin.ch/site/f/aussenpolitik/internationale.html

Euforic, site de l'Union européenne sur la coopération au développement: http://www.oneworld.org/euforic

OCDE, Comité d'aide au développement (CAD) : http://www.oecd.org/dac/ Ce site permet de se connecter à ceux des services de coopération des pays membres du CAD.

OFAEE: http://www.admin.ch/bawi/

Commande des publications de la DDC: DDC, Médias et communication, 3003 Berne, fax 031/324.13.48, e-mail: info@deza.admin.ch

\section{Aide humanitaire}

DDC, Corps suisse d'aide en cas de catastrophe (ASC) (brochure de présentation réalisée à l'occasion du 25e anniversaire de la création de l'ASC), DDC, Berne, 1998, 64 p.

DDC Swiss Commitment in Former Yugoslavia, DDC, Bern, August 1998.

DDC, Un seul monde (magazine), n³, septembre 1998 (dossier sur la Bosnie et la paix).

\section{Banques régionales de développement}

Asian Development Bank, Annual Report 1997, Manila, Philippines, 1998.

Banque interaméricaine de développement, Rapport annuel 1997, Washington, 1998.

L'Agefi, 27.3.98, 22.4.98, 27.4.98, 4.5.98 (assemblée annuelle de la Banque asiatique de développement).

Neue Zürcher Zeitung, «39. Jahresversammlung der IDB», 19.3.98.

\section{SITES INTERNET}

Banque asiatique de développement: http://www.adb.org

Banque africaine de développement: http://www.afdb.org

Banque interaméricaine de développement: http://www.iadb.org 